\title{
Semantic Interoperability of Management Information Modeling for Software-Defined Networking using a Formal Approach
}

\author{
Hui Xu, Chunzhi Wang and Xinlu Zong \\ School of Computer Science, Hubei University of Technology, Wuhan, China \\ xuhui@mail.hbut.edu.cn
}

\begin{abstract}
In Software-Defined Networking (SDN) domain, both SDN management and non-SDN network management co-exist, and may possibly integrate with each other in a unified manner. Thus in this interoperable network management scenario for SDN, many different meta-schemas might be utilized for information modeling of the same managed resource. This paper then tries to study the semantic interoperability problem of information modeling for SDN management from a formal viewpoint at the meta-schema level, introduces the thinking of extension and proposes a formal approach integrating the extension theory with the theory of concept lattices. Finally, case study validates that, proposed formal approach is feasible for semantic interoperability of management information modeling in SDN domain.
\end{abstract}

Keywords: Software-Defined Networking, management plane, information modeling, semantic interoperability, formal approach

\section{Introduction}

Software-Defined Networking (SDN) decouples the control plane and the data plane, and realizes the programming control with the use of SDN controllers. However, as for SDN, it seems that, besides the control plane and the data plane, an additional management plane is also required [1].

Open Networking Foundation (ONF) has already proposed and developed the OpenFlow Protocol for standardizations of SDN and the OpenFlow Management and Configuration Protocol (OF-CONFIG, the newest version is 1.2 up to now) [2] oriented to SDN management. ONF also makes use of its services area to work on technical projects to enable applications and network operator services with SDN technologies. Under this background, the information modeling project in ONF services area is responsible for a core information model that can be used as the basis for developing application-specific and forwarding technology-specific information models by other ONF projects in all ONF areas and external Service Data Objects (SDOs) [3].

On one side, YANG [4] is utilized by OF-CONFIG 1.2 for information modeling of SDN management. On the other side, both SDN management and non-SDN network management still co-exist, and may possibly integrate with each other in a unified manner [5-7]. Thus in this case, management information modeling calls for semantic interoperability. The aim of this paper is then to discuss the semantic interoperability problem of information modeling for SDN management, and propose a formal approach for the research on semantic interoperability of management information modeling in SDN domain.

The remainder of this paper is organized as follows. Section 2 discusses SDN management and its information modeling issues. Taking both the theory of concept lattices and the extension theory into considerations, Section 3 then proposes a formal approach to establish the basis of semantic interoperability for the integration of information models for SDN management. Section 4 provides case study in order to 
validate the feasibility of proposed formal approach for semantic interoperability of management information modeling in SDN domain. Section 5 concludes this paper.

\section{SDN Management and Its Information Modeling Issues}

As for the SDN management plane, it has following three implementation styles [1].

The first style is to add some network management functions to SDN controller, in other words, to implement the integration of control and management for SDN. The advantage of this implementation lies in low cost, high-level integration and rapid application. However, the main disadvantage is that, the management function of current OpenFlow protocol is limited. This limitation may be made up with existing management protocols, but the management problems of the SDN controller itself can not be solved.

The second style is to separate the SDN management function with the SDN control function, and to apply SDN management as a management approach for SDN. In this implementation, all network devices have a management interface to connect to the integrated management platform. The main advantage of this implementation is the reuse of existing management platforms, ensuring external monitoring and management of the SDN controller. But the main problem lies in the lack of specifications for communication manners and functions of SDN management plane and SDN control plane.

The third style is to separate the SDN management platform with the SDN control platform, and to realize distributed management. Advantages of this style include highreliability of the management plane, rapid reaction of events, and low-complexity of key management platform. However, this implementation requires not only communication of management messages between the controller on the control plane and the switches on the data plane, but also communication of management messages between the switches. Thus in this case, how to define appropriate management functions remains an important problem.

Furthermore, there are two models for integration of SDN and non-SDN network management, with explanation of how SDN controllers and non-SDN network management framework interact with each other, so as to maintain a reliable, end-to-end, user-oriented new generation Internet environment [7]. One is based on SDN that integrates with non-SDN network management, and the other one is based on nonSDN network management that integrates with SDN management.

The development of SDN management does favor to the research on its information modeling. And OF-CONFIG 1.2 has a companion YANG module for implementation of the OF-CONFIG data model. However, the SDN management plane still lacks a mature solution, and coexistence of SDN and non-SDN network management in new generation Internet environment provides an interoperable network management scenario.

Thus in this case, many different meta-schemas may possibly then be used for information modeling of the same managed resource, and the semantic interoperability problem of information modeling for SDN management is of great significance. In order to solve this problem, a formal viewpoint is seriously considered at the meta-schema level, so as to establish the basis of semantic interoperability of information modeling for SDN management.

\section{Proposed Formal Approach for Semantic Interoperability}

As one origin of Granular Computing $(\mathrm{GrC})$, concept lattices can be used to formally describe meta-schemas for management information modeling, and the theory of concept lattices that is Formal Concept Analysis (FCA) [8] prospects a promising way to information modeling for SDN management. According to ISO704: 2009 [9], terminology can be specified in different levels, which are the Object level, the Concept level and the Representation level. Based on this classification, taking the theory of concept lattices into consideration, information modeling for SDN management can be 
divided into three levels, which are the Formal Context level, the Formal Concept level, and the Formal Representation level. Starting from the basic Formal Context level, this section then proposes a formal approach based on the extension theory combined with the theory of concept lattices for semantic interoperability of information modeling in SDN domain.

\subsection{Possible Conflicts when Obtaining Formal Contexts}

By applying the theory of concept lattices, Definition 1 provides a basic specification of formal contexts of information modeling for SDN management at the meta-schema level.

Definition 1 Suppose that, a) entities for modeling SDN management information are specified as $E$, b) attributes of these entities are specified as $A$, and c) if $e \in E, a \in A,(e, a) \in R$, then it means that $R$ represents the relationships between $E$ and $A$,namely $e R a$, thus in this way, a basic formal context of meta-schema information modeling for SDN management is defined as $I=(E, A, R)$.

However, existing meta-schemas may possibly adopt correlated manners of information modeling for the interoperable network management scenario in SDN domain. For example, "organization", "status", "description" and "reference" from YANG have respectively a same semantic meaning with "ORGANIZATION", "STATUS", "DESCRIPTION" and "REFERENCE" from Structure of Management Information version 2 (SMIv2) [10], without conflicts in naming terms.

Thus then, the following two main conflicts must be seriously considered when obtaining formal contexts that include entities, attributes and their relations, from metaschemas used in the interoperable network management scenario for SDN.

a) Synonymy conflicts

Synonymy conflicts occur when a same entity or attribute defined by different terms. Although in OF-CONFIG 1.2, the YANG language acts as the meta-schema for information modeling of SDN management, but both SDN management and non-SDN network management still co-exist, and other meta-schemas may possibly be used in information modeling for SDN management. Thus in this case, the same entity or the same attribute from the semantic viewpoint may be described in different formats.

Figure 1 and Figure 2 show respectively two managed objects with the same semantic meaning used in interface management, which are "ifIndex" defined by SMIv2 for nonSDN network management, and "if-index" defined by YANG possibly for SDN management.

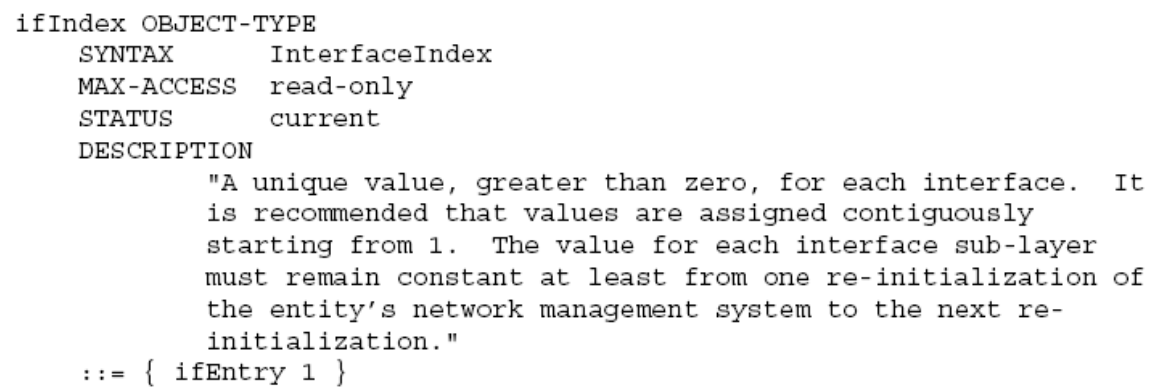

Figure 1. An Example from the Smiv2-Defined MIB for Interface Management 


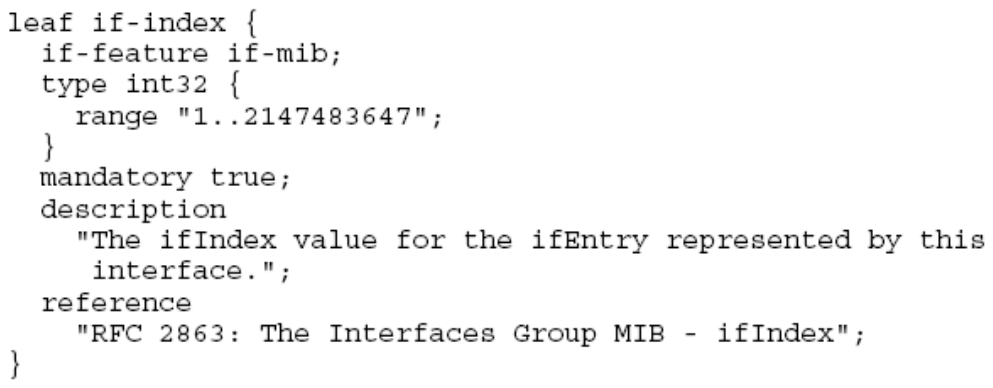

Figure 2. An Example From the YANG Data Model for Interface Management

As is indicated in Figure 1 and Figure 2, SMIv2 and YANG use different formats, which are "OBJECT-TYPE" and "leaf" respectively, to define two semantically-same managed objects in the form of different terms.

b) Coverage conflicts

Coverage conflicts exist when two correlated entities or attributes that one has a broader domain than the other one. Figure 3 and Figure 4 show two examples respectively using "CHOICE" from SMIv2 and "choice" from YANG.

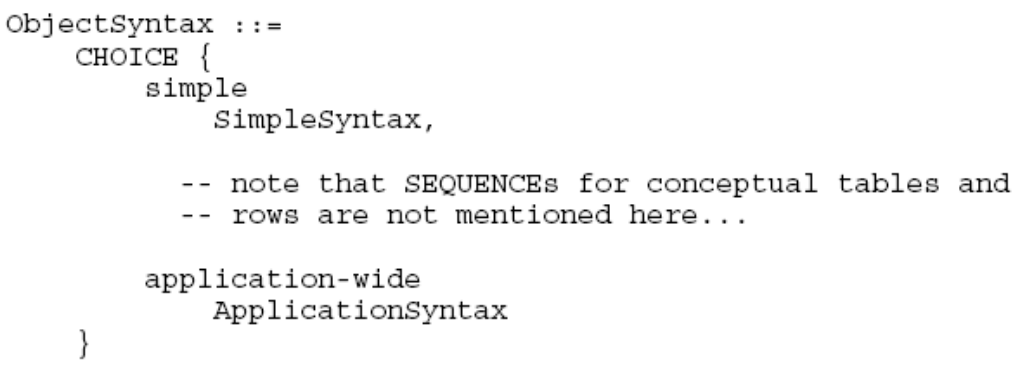

Figure 3. An Example Using "CHOICE" from SMlv2 


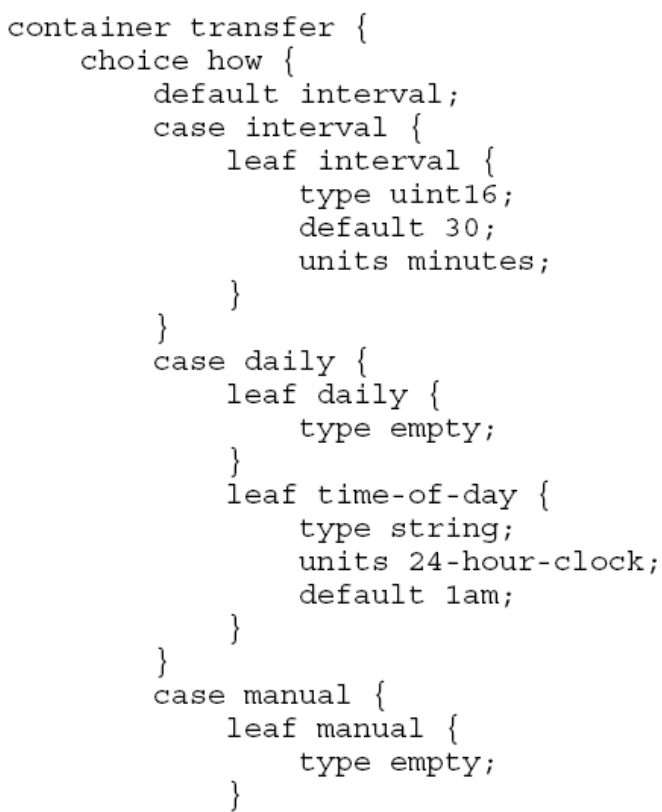

Figure 4. An Example Using "Choice" from YANG

As is demonstrated in Figure 3 and Figure 4, both "CHOICE" from SMIv2 and "choice" from YANG use a same naming term to define that, only one of the alternatives may occur at any one time. But from a semantic viewpoint, YANG adopts a broader domain for "choice" than that of "CHOICE" from SMIv2, as revealed in Figure 5.

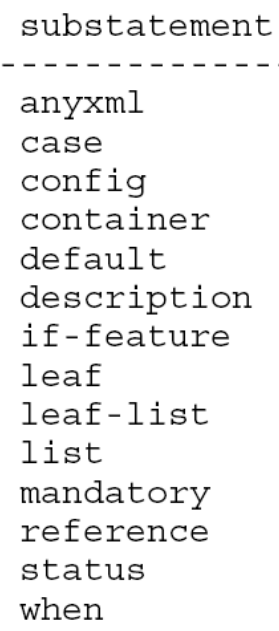

Figure 5. A Broader Domain for "Choice" from YANG

\subsection{Optimized Formal Contexts with the Use of Matter-Elements}

The key reason for these conflicts stated above lies in the fact that, the formal contexts based on the theory of concept lattices usually use a term to identify an entity or an attribute. Since existing meta-schemas may possibly adopt correlated manners of information modeling for an interoperable network management scenario in SDN domain, 
a simple term may be not enough to uniquely distinguish entities or attributes in most cases. The thinking of extension is then introduced into the research for this case, and the logic cell of the extension theory is basic-elements [11], one kind of which is matterelements.

Formula 1 proposes a specification of matter-elements for extension of entities, in which $\operatorname{Term}_{x}$ means an identifier of an entity for modeling SDN management information, with its characteristics $e_{1}, e_{2}, \ldots, e_{m}$ and corresponding values $p_{1}, p_{2}, \ldots, p_{m}$. And the class for this kind of matter-elements is defined as Formula 2.

$$
\begin{array}{r}
M_{e}=\left[\begin{array}{c}
\text { Term }_{x}, e_{1}, p_{1} \\
\ldots \\
e_{m}, p_{m}
\end{array}\right]=\left(\text { Term }_{x}, e, p\right) \\
\left\{M_{e}\right\}=\left[\begin{array}{c}
\left\{\text { Term }_{x}\right\}, e_{1}, p_{1} \\
\ldots \\
e_{m}, p_{m}
\end{array}\right]=\left(\left\{\text { Term }_{x}\right\}, e, p\right)
\end{array}
$$

In a similar way, definitions of matter-elements for extension of attributes and the class for this kind of matter-elements are respectively demonstrated in Formula 3 and Formula 4.

$$
\begin{array}{r}
M_{a}=\left[\begin{array}{r}
\text { Term }_{y}, a_{1}, q_{1} \\
\ldots \\
a_{n}, q_{n}
\end{array}\right]=\left(\text { Term }_{y}, a, q\right) \\
\left\{M_{a}\right\}=\left[\begin{array}{r}
\left\{\text { Term }_{y}\right\}, a_{1}, q_{1} \\
\ldots \\
a_{n}, q_{n}
\end{array}\right]=\left(\left\{\text { Term }_{y}\right\}, a, q\right)
\end{array}
$$

Using Formula 1-4 based on the extension theory, Definition 2 proposes an optimized specification of formal contexts of information modeling for SDN management.

Definition 2 Suppose that, a) an entity for modeling SDN management information is extended as a matter-element $\left.M_{e}=\left(\operatorname{Term}_{x}, e, p\right), \mathrm{b}\right)$ an attribute of the entity is extended as a matter-element $M_{a}=\left(\operatorname{Term}_{y}, a, q\right)$, and c) if $e \in M_{e}, a \in M_{a},(e, a) \in R$, then it means that $R$ represents the relationships between $M_{e}$ and $M_{a}$,namely $e R a$, thus in this way, an optimized formal context of information modeling for SDN management at the meta-schema level by means of extension can be defined as $I^{\prime}=\left(\left\{M_{e}\right\},\left\{M_{a}\right\}, R\right)$.

\subsection{Extension Principles for Semantic Interoperability}

When Definition 2 is used for obtaining formal context of management information modeling in SDN domain at the meta-schema level, extension principles can then be utilized to semantic interoperability of information modeling for SDN management, examples of which are as follows.

For the sake of semantic interoperability, Principle 1 presents a divergence principle to integrate different formats in other meta-schemas for entities of the same semantic meaning, while Principle 2 demonstrates a divergence principle to integrate different formats in other meta-schemas for attributes of the same semantic meaning. 
Principle 1 As for a matter-element $M_{e}=\left(\right.$ Term $\left._{x}, e, p\right)$ representing an entity from the formal context for modeling SDN management information, different terms term $_{x_{-} i}(1 \leq i \leq k)$ for the same semantic meaning of ${ }^{M_{e}}$ can be extended as

$$
M_{e}=\left(\operatorname{Term}_{x}, e, p\right)-\mid\left\{\begin{array}{l}
\left(\text { Term }_{x_{-} 1}, e, p\right), \\
\left(\text { Term }_{x_{-} 2}, e, p\right), \\
\ldots, \\
\left(\text { Term }_{x_{-} k}, e, p\right)
\end{array}\right\}
$$

Principle 2 As for a matter-element $M_{a}=\left(\operatorname{Term}_{y}, a, q\right)$ representing an attribute from the formal context for modeling SDN management information, different terms $\operatorname{Term}_{y_{-} j}(1 \leq j \leq l)$ for the same semantic meaning of ${ }^{M_{a}}$ can be extended as

$$
M_{a}=\left(\operatorname{Term}_{y}, a, q\right)-\mid\left\{\begin{array}{l}
\left(\operatorname{Term}_{y_{-} 1}, a, q\right), \\
\left(\operatorname{Term}_{y_{-} 2}, a, q\right), \\
\ldots, \\
\left(\operatorname{Term}_{y_{-} l}, a, q\right)
\end{array}\right\}
$$

When applying Principle 1 and Principle 2, different terms from other meta-schemas for the same semantic meaning can be integrated into current entity or attribute by means of extension, so as to avoid synonymy conflicts. Thus in this way, the thinking of extension can be utilized to meta-schema merging of management information modeling in SDN domain, for the sake of semantic interoperability.

\section{Case Study}

In order to validate the feasibility of proposed formal approach for semantic interoperability of management information modeling in SDN domain, this section take the attribute "default" from YANG as an example. According to Formula 3, extension of its corresponding attribute in formal contexts for management information modeling in SDN domain can be defined as the following matter-element.

$$
M_{\text {default }}=\left[\begin{array}{c}
\text { "default", semantic_meaning }, a_{-} \text {default_value } \\
\text { necessary, } n o
\end{array}\right]
$$

And in SMIv2, there is the DEFVAL clause with the same semantic meaning. Thus, considering semantic interoperability for management information modeling in SDN domain, meta-schema merging is greatly required. With the use of Principle 2, extension by means of divergence is applied to integrate the term "default" from YANG with the term "DEFVAL" from SMIv2 for attributes of the same semantic meaning, shown as follows.

$$
\begin{aligned}
M_{\text {default }} & =\left[\begin{array}{c}
\text { default", semantic_meaning, } a_{-} \text {default_value } \\
\text { necessary,no }
\end{array}\right] \\
-\mid M_{D E F V A L} & =\left[\begin{array}{c}
\text { DEFVAL", semantic_meaning, a_default_value } \\
\text { necessary, no }
\end{array}\right]
\end{aligned}
$$

Then, extension of corresponding attributes for the term "default" from YANG and the term "DEFVAL" from SMIv2 in formal contexts for management information modeling in SDN domain can be semantically merged as a class for this kind of matter-elements as follows, according to Formula 4. 


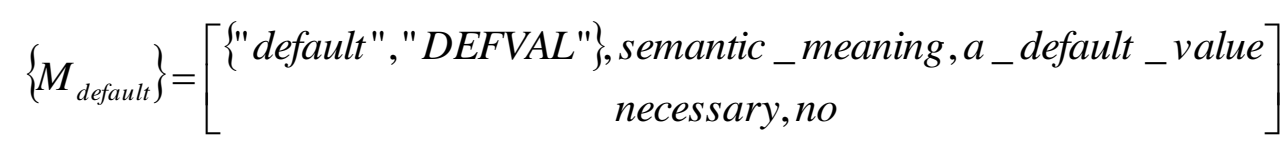

In summary, case study shows that, proposed formal approach is feasible for semantic interoperability of management information modeling in SDN domain.

\section{Conclusions}

The main contribution of this paper is to introduce the thinking of extension into the study on semantic interoperability for management information modeling in SDN domain, and propose a formal approach based on the extension theory integrating with the theory of concept lattices.

\section{Acknowledgements}

This work has been supported by the National Natural Science Foundation of China (No. 61602162, No. 61440024), and the Doctoral Scientific Research Fund from Hubei University of Technology (No. BSQD12029). The authors would like to thank all project partners for their valuable contributions and feedbacks.

\section{References}

[1] S. Kuklinski and P. Chemouil. Network Management Challenges in Software-Defined Networks. IEICE Transactions on Communications, Vol. E97-B (2014), No. 1, pp. 2-9

[2] Open Networking Foundation. OpenFlow Management and Configuration Protocol 1.2 (OF-CONFIG 1.2). Available: www.opennetworking.org/technical-communities/areas/specification/1928-of-config (2015)

[3] Open Networking Foundation. Information Modeling Project for ONF Services Area. Available: www.opennetworking.org/technical-communities/areas/services (2015)

[4] M. Bjorklund ed. YANG - A Data Modeling Language for the Network Configuration Protocol (NETCONF). RFC6020 (2010)

[5] F. N. N. Farias, J. J. Salvatti, E. C. Cerqueira and A. J. G. Abelem. A Proposal Management of The Legacy Network Environment using Openflow Control Plane. Proceedings of 13th IFIP/IEEE Network Operations and Management Symposium (2012), pp. 1143-1150

[6] N. Koutsouris, K. Tsagkaris, P. Demestichas, L. Mamatas, S. Clayman and A. Galis. Managing Software-Driven Networks with a Unified Management Framework. Proceedings of 13th IFIP/IEEE International Symposium on Integrated Network Management (2013), pp. 1084-1085

[7] D. Kim, J. M. Gil, G. Wang and S. H. Kim. Integrated SDN and Non-SDN Network Management Approaches for Future Internet Environment. Lecture Notes in Electrical Engineering, Vol. 240 (2013), pp. 529-536

[8] B. Ganter and R. Wille. Formal Concept Analysis: Mathematical Foundations. Springer Press, Berlin (1999)

[9] International Organization for Standardization. Terminology work - Principles and methods. ISO704 (2009)

[10] K. McCloghrie Structure of Management Information Version 2 (SMIv2). RFC2578 (1999).

[11] W. Cai, C. Y. Yang and B. He. Preliminary Extension Logic. Science Press, Beijing (2003), Simplified Chinese version 


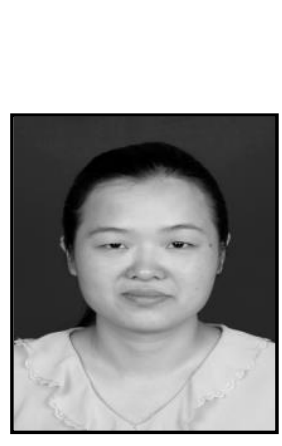

\begin{abstract}
Authors
Hui Xu, She received a bachelor's degree in Computer Science and Technology from Huazhong Normal University, Wuhan, China in 2005, a master's degree in Computer Application Technology from Huazhong Normal University, Wuhan, China in 2008, and a doctor's degree in Radio Physics from Huazhong Normal University, Wuhan, China in 2010. Since 2006, she has been a certified computer system analyst in China. Now, she is an Associate Professor at the School of Computer Science in Hubei University of Technology, Wuhan, China. Currently, her major field of study is network and service management. Dr. Xu became a Member of Institute of Electrical and Electronics Engineers (IEEE) in 2007, a Member of Association for Computing Machinery (ACM) in 2007 and a Member of China Computer Federation (CCF) in 2008. She has authored or coauthored 1 book and 2 book chapters in the field of network management, about 10 papers published by Chinese journals, more than 10 papers published by international journals, and more than 20 papers published by international conferences. In April 2008, she was awarded by International Association of Engineers (IAENG) for her first-authored paper presented to 2008 IAENG International Conference on Communication Systems and Applications. Additionally, she was a Session Co-Chair or a Paper Reviewer for 2nd\&3rd\&7th\&8th International Conference on Computer Science and Education (ICCSE 2007\&2008\&2012\&2013), a Session Chair for 1st International Symposium on Electronic Commerce and Security (ISECS 2008), a Paper Reviewer for 4th IEEE Conference on Industrial Electronics and Applications (ICIEA 2009), a Paper Reviewer for 3rd International Conference on Computer and Network Technology (ICCNT 2011), a Paper Reviewer for 32nd Chinese Control Conference (CCC 2013), and a Paper Reviewer for Security and Communication Networks, an international journal published by Wiley Press.
\end{abstract}

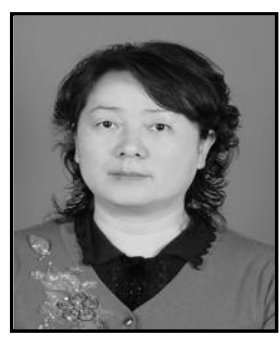

Chunzhi Wang, She is a Professor at the School of Computer Science in Hubei University of Technology, Wuhan, China. She is also the Dean of the School of Computer Science in Hubei University of Technology, Wuhan, China. Currently, her major field of study is Software-Defined Networking.

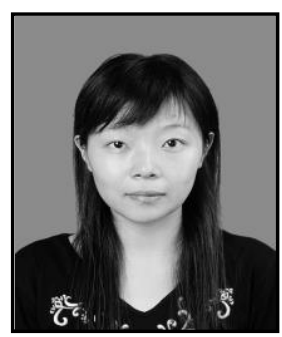

Xinlu Zong, She is a Lecturer at the School of Computer Science in Hubei University of Technology, Wuhan, China. Currently, her major field of study is automated management. 
International Journal of Control and Automation Vol.10, No.4 (2017) 